\title{
Comparative efficacy of inspiratory, expiratory and combined respiratory muscle training on the pulmonary functions and chest expansion in acute stroke survivors
}

\author{
Adeogun Abiodun $\mathrm{A}^{1 *}$ and Umar Dolapo $\mathrm{K}^{2}$ \\ ${ }^{1}$ Principal Physiotherapist, Department of Physiotherapy, General Hospital Odan Lagos, Nigeria \\ ${ }^{2}$ Physiotherapist/Clinical Research Officer, Department of Physiotherapy, General Hospital Odan \\ Lagos, Nigeria
}

\section{Abstract}

Background: Respiratory muscle strength can be reduced in patients diagnosed with stroke, which reasonably justifies the use of respiratory muscle training in this population. This study determines the comparative efficacy of inspiratory, expiratory, and combined respiratory muscle training on the pulmonary functions and chest expansion in acute stroke survivors.

Method: Forty-five acute stroke survivors (15 in each group) completed all protocols of the study. Participants were randomly assigned to any one of three groups. In addition to the conventional exercise therapy, participants received any one of the three respiratory muscle training protocols (inspiratory muscle training, expiratory muscle training or combined respiratory muscle training). Chest expansion was assessed using tape measure and pulmonary function parameters were assessed using a spirometer.

Results: Paired t-test analysis showed significant improvements in the chest expansion and the pulmonary function parameters following training in each group. One-way ANOVA showed significant improvements in the pulmonary function parameters across the three groups but not in the chest expansion with $p$ - value $=0.405$. Least significant difference (LSD), post-hoc analysis shows that the significant difference for $\mathrm{FEV}_{1}, \mathrm{FVC}$ and $\mathrm{FEV}_{1} / \mathrm{FVC}$ lies between inspiratory muscle training group and expiratory muscle training group.

Conclusion: When the three training methods were compared, it was found that expiratory muscle training was the most beneficial in improving the pulmonary functions and chest expansion in acute stroke survivors.

\section{Introduction}

Impaired motor function is one of the most frequent and persistent consequences of stroke [1]. Not only are peripheral muscles involved in poststroke disability, but there are also respiratory muscle weakness, low thoracic expansion, and postural trunk dysfunction [2]. These may also play an important role in exercise capacity and the ability to carry out activities of daily living [3-7]. Intolerance to exercise in patients diagnosed with stroke may be due to respiratory muscle impairment, resulting from decreased lung volumes and decreased inspiratory and expiratory strength [2].
More Information

*Address for Correspondence:

Adeogun Abiodun A, Principal Physiotherapist, Department of Physiotherapy, General Hospital Odan Lagos, Nigeria, Tel: +2348033319845; Email: ptadeogun@gmail.com; akanbi. adeogun@lagosstate.gov.ng

Submitted: June 30, 2021

Approved: July 15, 2021

Published: July 16, 2021

How to cite this article: Adeogun Abiodun A, Umar Dolapo K. Comparative efficacy of inspiratory, expiratory and combined respiratory muscle training on the pulmonary functions and chest expansion in acute stroke survivors. J Nov Physiother Rehabil. 2021; 5: 010-018.

DOI: 10.29328/journal.jnpr.1001040

Copyright: () 2021 Adeogun Abiodun A, et al. This is an open access article distributed under the Creative Commons Attribution License, which permits unrestricted use, distribution, and reproduction in any medium, provided the original work is properly cited.

Keywords: Acute stroke survivors; Respiratory muscle training; Chest expansion; Pulmonary functions

D) Check for updates

OPEN ACCESS
Respiratory muscle training has been shown to have positive effects on the pulmonary functions and exercise tolerance, which ultimately can help patients carry out their activities of daily living more easily $[1,8]$.

Acute stroke survivors had decreased respiratory muscle strength and consequent diaphragm and abdominal dysfunction $[9,10]$. Studies have shown that acute stroke patients also show decreased respiratory function [9,11]. Khedr [12] reported decreased diaphragmatic excursion in $41 \%$ of patients and reduced FVC and $\mathrm{FEV}_{1}$, as much as $50 \%$ of values predicted for unaffected individuals. Tomczak [13] also 
demonstrated that acute stroke survivors presented lower values of FVC, $\mathrm{FEV}_{1}$, and tidal volume when compared with predictive values, which justifies the use of respiratory muscle training in this group of patients. Respiratory muscle training resulted in an increased $\mathrm{FEV}_{1}$ and FVC. This improvement can be associated with increased respiratory muscle strength.

Stroke survivors often have poor lung function, which has been found to be approximately $50 \%$ below that of age- and sex-adjusted norms $[14,15]$. Post-stroke pneumonia (PSP) is a well-known complication during the first few weeks after stroke [16], and the incidence ranges from $2 \%$ to $57 \%$ in different studies, with a median of $10 \%$ [16]. Patients who develop PSP have higher mortality, longer hospitalization, worse functional outcomes, and higher care needs [16,17]. Stroke impairs respiratory muscle strength and cough flow by approximately 50\% compared with non-stroke controls $[4,6]$. Respiratory muscle weakness is attributable to the disruption of central motor output, as opposed to the intrinsic loss of peripheral muscle function [4]. Impaired swallow and increased risk of aspiration are major risk factors for PSP [17]. A cough (voluntary or reflex) is the most immediate protective mechanism from aspiration [18] and requires coordinated activation of inspiratory, expiratory, and intrinsic laryngeal muscles [19].

Reduced respiratory muscle strength has been observed in patients suffering from a stroke but little attention has been paid to it, possibly because it is often not associated with any obvious symptoms $[6,20]$. Generally, respiratory muscle weakness is attributed to the impairment of the muscles involved in respiration, induced by the central nervous system (CNS) lesions [11]. The cardiopulmonary functions of patients with hemiplegia caused by stroke diminish due to the decreased expansion of the damaged hemi-thorax and deterioration of the respiratory muscles. Furthermore, the common symptoms observed in stroke patients, including ankylosis of joints and limited use of muscular movement, result in a decrease in cardiorespiratory control and the capacity of oxygentransferring systems due to a combined lack of oxygen and increased metabolic demands [7].

The thorax cannot expand sufficiently due to the paralysis of the diaphragm and respiratory muscles following a stroke. Continuation of this condition can lead to shortening of the thoracic muscles and muscle fibrosis. Consequently, the level of thoracic expansion may be reduced during breathing [21]. Therefore, deterioration of the pulmonary functions and reduced chest expansion are significant factors associated with stroke patients and as such requires intensive rehabilitation treatment [22].

A general stroke rehabilitation program, which focuses only on physical and functional recovery, may not sufficiently enhance the patient's cardiopulmonary function [14,23]. Therefore, a rehabilitation program integrated with breathing-related intervention will be effective in improving the pulmonary functions and the ability to perform functional activities in patients with stroke [24,25].

Inspiratory muscle training (IMT) for improvement in respiratory function involves the application of a load to the diaphragm [26]. According to previous studies, IMT can improve maximal inspiratory pressure (MIP), lung function, endurance, and exercise performance after 6 to 8 weeks of training [21,27]. According to St Croix [28], it was reported that respiratory metabolism is reflected in exercise performance. Fatigue in respiratory muscles can be reduced or delayed by strengthening; this improves exercise capacity and respiratory movement, which are necessary for improvement in functionality [29]. The use of inspiratory muscle training device for one year in a cohort study of 42 patients with COPD improved respiratory muscle strength and exercise capacity, reduced dyspnea and days of hospitalization, and improved the overall quality of everyday life [30]. Inspiratory muscle training was also used for twelve weeks in a study of ten (10) patients with Parkinson disease, showing improvement in respiratory muscle strength, increased endurance, and enhancement of dyspnea and cognitive ability [31].

Expiratory muscle training (EMT) elicits similar responses to IMT in the expiratory muscle system, although much less data is available to date, compared to evidence on the effect of IMT. Akin to inspiratory muscle training, improvement of the maximal expiratory pressure (MEP) is the hallmark parameter of successful EMT. Interestingly, EMT alone also leads to improved maximal inspiratory pressure, demonstrating the involvement of the inspiratory muscles in the process of expiration, whereas IMT does not improve maximal expiratory pressure [32]. Due to the importance of expiratory muscles in speech and swallow functions, EMT is of particular interest for patients with dysphonia, dysphagia, and reduced ability for airway clearance, such as those with Parkinson's disease or other neuromuscular diseases (NMDs).

While benefits of either IMT or EMT alone are clearly demonstrated in the literature, combination of IMT and EMT has not been widely reported. However, a few studies highlight the possibly overlooked potential of combined IMT and EMT. A 4-week study combined inspiratory exercises and expiration with pursed lips, which significantly improved Tiffeneau index $\left(\mathrm{FEV}_{1} /\right.$ Vital Capacity), total volume, and inspiratory capacity in stroke patient [33]. Combined IMT and EMT in patients with Duchenne muscular dystrophy or spinal muscular atrophy improved inspiratory and expiratory muscle strength and led to a sustained reduction of respiratory load perception, thus improving patient comfort and HealthRelated Quality of Life (HRQoL) [34]. In patients with multiple sclerosis, combined IMT/EMT improved maximal inspiratory and expiratory pressure, and significantly reduced fatigue [35]. 
Most importantly, direct comparison of the effects of IMT, EMT or a combination thereof on the pulmonary functions has not been reported in stroke patients except in COPD patients where maximal inspiratory and expiratory pressure improved by $33 \%$ when inspiratory and expiratory muscles were trained simultaneously, but only by $20 \%-25 \%$ when only one set of muscles was strengthened [36]. These findings clearly suggest that a combination of IMT and EMT may be more effective than either method alone, in improving pulmonary functions. These findings clearly suggest that a combination of IMT and EMT may be more effective than either method alone, in improving pulmonary functions. Hence, this study is designed to compare the efficacy of inspiratory, expiratory, and combined respiratory muscle training on the pulmonary functions and chest expansion in acute stroke survivors.

\section{Materials and methods}

\section{Study design}

This study was a single-blind randomized controlled trial. This study is registered with the South African Medical Research Council (Pan African Clinical Trial Registry) with unique identification registry number - PACTR201711002738361.

\section{Determination of sample size}

The sample size calculation was done based using Cohen's standard effect size [37] and determined as a minimum of 15 subjects in each group using the single mean sample size formula with $\alpha$ set at 0.05 and $\beta$ at 0.20 .

\section{Ethical consideration}

Participants were recruited from the neurology unit through referrals to the physiotherapy (in-patient) department of General Hospital Odan, Lagos, Nigeria. Prior to the commencement of this trial, ethical approval was sought and obtained from the Health Research and Ethics Committee of the Lagos University Teaching Hospital (LUTH) and the Health Research and Ethics Committee of the General Hospital Odan, Lagos through the Lagos State Health Service Commission (HSC).

\section{Study participants}

A total of sixty-two (62) patients diagnosed with acute stroke (onset of 2 weeks) whose blood pressures were controlled, and those willing to participate in the study were included. Patients with acute stroke who had signs of increased intracranial pressure on CT-Scan, poorly controlled hypertension (blood pressure higher than 180/100 $\mathrm{mmHg}$ on three or more occasions over the preceding 24 hours), myocardial infarction, angina, or acute heart failure in the preceding 3 months, pulmonary, neurologic, or orthopaedic conditions adversely affecting the respiratory pump, including asthma and chronic obstructive pulmonary disease (COPD) were excluded from the study. Informed consent was obtained from the participants before the commencement of the trial and confidentiality of the data were assured. The participants were screened for eligibility based on the inclusion and exclusion criteria. Fifty-one (51) of them met the criteria and eleven (11) were excluded from the study. The fifty-one participants were randomly assigned to three groups using computer generated random number sequence. Subjects in Group-A received conventional exercise therapy and inspiratory muscle training, Group-B received conventional exercise therapy and expiratory muscle training and, Group-C received conventional exercise therapy and combined respiratory muscle training (Figure 1).

\section{Outcome measures}

A procedural checklist and standardized verbal instructions followed by demonstrations was used to ensure the uniformity of the procedures during the clinical study. In this study, the participants were unaware of the test results and interventional groupings. The following were obtained from the participants: demographic characteristics including age, weight, height, and body mass index (BMI). The outcome variables were measured two times, within 24 hours after referral for physiotherapy (baseline values), and at $4^{\text {th }}$ week post-intervention. This measurement of the chest expansion using a tape measure, measurement of pulmonary functions $\left(\mathrm{FEV}_{1}, \mathrm{FVC}, \mathrm{FEV}_{1} / \mathrm{FVC}\right.$ and $\left.\mathrm{PEF}\right)$ using a spirometer.

\section{Measurement of chest expansion}

To measure chest expansion when breathing, a participant's chest wall was measured in an upright sitting position with a tape measure. This was done in a cubicle to ensure privacy.

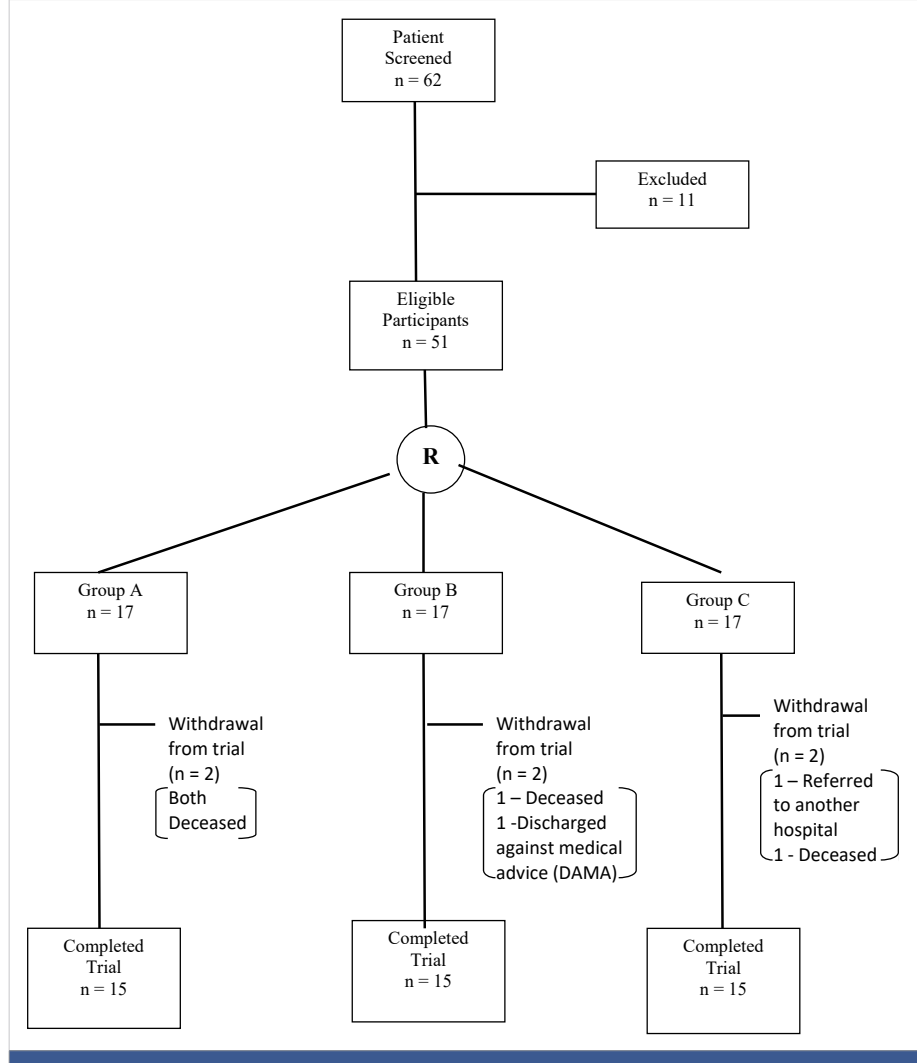

Figure 1: CONSORT diagram of the study. R: Randomization. 
The chest wall was exposed, and the circumference was measured at maximal inspiration, and maximal expiration. The tape measure was placed over the areas that connect the xiphoid process and the corpus sterni. The extent of chest expansion was calculated by determining the differences in circumferences of the chest wall in a state of maximal expiration and maximal inspiration [38]; and the mean value of three measurements was recorded [39]. It was measured in centimetres $(\mathrm{cm})$.

\section{Measurement of pulmonary functions}

All pulmonary function measurements (using spirometer) were taken with the participants in the sitting position. The participant was instructed to inhale deeply and exhale into the mouthpiece of the spirometer, and the mean value of three measurements was recorded [39]. This study measured forced vital capacity (FVC), forced expiratory volume in one second $\left(\mathrm{FEV}_{1}\right)$, and the $\mathrm{FEV}_{1} / \mathrm{FVC}$ ratio, to determine signs of obstructive or limited pulmonary disease such as strokeassociated pneumonia. The peak expiratory flow (PEF) was measured to assess resistance in the respiratory tracts.

\section{Assessment of respiratory muscle strength}

The maximum inspiratory pressure (MIP) and maximum expiratory pressure (MEP) were predicted in the participants using the reference equations below; Male MIP reference = 120 - (0.41 x Age); Female MIP reference = $108-(0.61$ x Age); Male $\mathrm{MEP}$ reference $=174-(0.83 \times$ Age $)$; Female MEP reference $=131-(0.86 \times$ Age $)[40]$.

\section{Intervention}

Prior to exercise training, participants in the three groups underwent a warm-up period. They had a cool-down period after each session of exercise training.

Warm-up period - participants were told to breathe in through the nostrils at maximum inspiration and breathe out through the pursed-lips. The participants were told to do two sets of 10 breaths. This was done to increase lung expansion. Passive mobilization was done to the affected upper limb and lower limb. The warm-up period was completed with stretching exercises to the muscles of the upper extremities, lower extremities, and the back [41].

Conventional exercise training - The three groups received the same conventional exercise treatments for 30 minutes (including joint mobility, eccentric contraction, and muscle strengthening exercises to the affected upper limb and lower limb), followed by a 10-minute rest [42].

Cool-down period - Participants were told to breathe in through the nostrils at maximum inspiration and breathe out through the pursed-lips. The participants were told to do two sets of 10 breaths. This was done to facilitate relaxation. Stretching exercises to the quadriceps, hamstrings, and calf muscles, to relax the muscles of the lower extremities. The cool-down period was completed with stretching the muscles in arms, neck, upper back, and shoulders to relax all the major muscle groups of the body [41].

\section{Group-A}

Participants in this group trained daily over a 4-week period. Participants performed five sets of 10 breaths with the POWERbreathe trainer, with resting periods of 1 minute between sets [43]. The training resistance was set at $50 \%$ of the individual's MIP. The participants were seated upright and holding the POWERbreathe trainer with the unaffected hand in the upright position. They were instructed to place the mouthpiece in the mouth so that the 'two wings' rest between the teeth and gums and then grip the two bits between the teeth to form an airtight seal. With the mouthpiece in place, the nose clip was used to hold the nostrils together so as not to breathe in through the nose. They were instructed to take a sharp breath in through the mouthpiece and continued to breathe in as hard as they could until the lungs were full (this took about 3 seconds). They were told to breathe out slowly through the mouthpiece until the lungs were completely empty, letting the muscles in the chest and shoulder to relax.

\section{Group-B}

Participants in this group trained daily over a 4-week period. Participants performed five sets of 10 breaths with the Ultrabreathe trainer, with resting periods of 1 minute between sets [43]. The training resistance was set at $50 \%$ of the individual's MEP. The Ultrabreathe trainer was used for expiratory muscle training, which exercises the muscles that are used for breathing out by simply turning the cap of the device clockwise as far possible to seal off the exhaust outlet. The participants were seated upright and holding the Ultrabreathe trainer with the unaffected hand in the upright position. They were instructed to place the mouthpiece in the mouth so that the 'two wings' rest between the teeth and gums and then grip the two bits between the teeth to form an airtight seal. They were instructed to breathe in through the nose and out through the mouthpiece, feeling the abdominal muscles contract (tighten) as they help to support in breathing out.

\section{Group-C}

Participants in this group trained daily over a 4-week period. Participants performed five sets of 10 breaths with the breather trainer, with resting periods of 1 minute between sets [43]. The training resistance was set at $30 \%$ of the individual's maximal mouth pressures (MIP and MEP) [44]. This group was taught combined IMT and EMT training. The participants were seated in an upright position, holding the breather trainer. The participants then place the mouthpiece of the breather trainer in the mouth so that the lips cover the outer shield to make a seal. The participants were instructed to inspire (breath in) deeply filling the lungs and holding the breath in the lungs for 2 to 3 seconds before exhaling forcefully while the breather trainer is still in place. 


\section{Statistical analysis}

Descriptive statistics of mean, standard deviation, bar chart and pie chart were used to summarize the demographics, paretic side of stroke affection and the type of stroke in each group. Paired t-test was used to determine the effects of each training on the chest expansion and the pulmonary function parameters pre-and post-training. One-way analysis of variance (ANOVA) was used to compare the differences in the chest expansion and pulmonary function parameters across the three groups post-training and post-hoc analysis was used to determine where the significant difference lies between groups. The level of significance was set at $p \leq 0.05$.

\section{Results}

\section{Demographic data}

Fifty-one (51) participants were eligible for the study after screening. They were divided into three groups with seventeen (17) participants in each group. However, only 15 participants in each group completed the study. They comprised 34 (75.6\%) male and 11 (24.4\%) females (Figure 2). Their ages ranged from 37 - 82 years. The age distribution of the participants is shown on table 1 . Sixteen (35.6\%) of them had their age ranged from $50-59$ years.

The mean ages of the participants in the three groups (A, B and C) were $56.20 \pm 10.39$ years, $59.27 \pm 12.98$ years, and $58.20 \pm 10.12$ years, respectively. The mean Body Mass Indices (BMI) of the participants in the three groups were $23.74 \pm 3.27 \mathrm{Kg} / \mathrm{m}^{2}, 25.92 \pm 3.31 \mathrm{Kg} / \mathrm{m}^{2}$ and $26.11 \pm 3.80 \mathrm{Kg} /$ $\mathrm{m}^{2}$ respectively (Table 2 ). There was no statistically significant difference in the physical characteristics of the participants in the three groups, that is, the three groups were homogenous.

Group B had eleven (73.3\%) of ischaemic stroke while group C had four (26.7\%) of haemorrhagic stroke (Figure 3). Seven $(46.7 \%)$ of the participants in group A had left hemiparesis while nine $(60 \%)$ of the participants in group B had right hemiparesis (Figure 4).

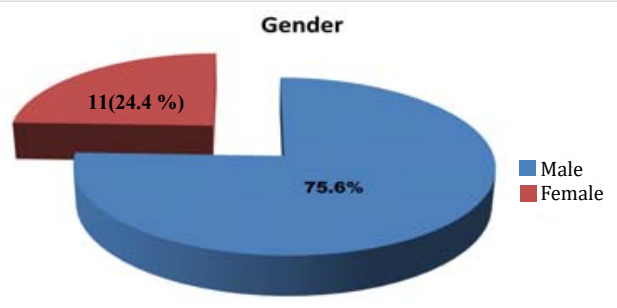

Figure 2: Pie-Chart showing the sex distribution of the participants.

Table 1: Age range distribution of the participants.

\begin{tabular}{|c|c|c|}
\hline Age range (years) & Frequency $(\mathbf{n})$ & Percentage (\%) \\
\hline $30-39$ & 1 & 2.2 \\
\hline $40-49$ & 10 & 22.2 \\
\hline $50-59$ & 16 & 35.6 \\
\hline $60-69$ & 11 & 24.4 \\
\hline $70-79$ & 6 & 13.3 \\
\hline $80-89$ & 1 & 2.2 \\
\hline Total & 45 & 100.0 \\
\hline
\end{tabular}

Pre - and post - intervention comparison of the chest expansion (CE) values and pulmonary function parameters within each group.

There were statistically significant differences in the chest expansion values and pulmonary function parameters of the participants in Group-A (CE: $p=0.001 ; \mathrm{FEV}_{1}: p=0.003$; FVC: $p=0.023 ; \mathrm{FEV}_{1}$ /FVC: $p=0.001$; PEF: $p=0.005$ ). There were statistically significant differences in the chest expansion values and pulmonary function parameters of the participants in Group-B (CE: $p=0.001 ;$ FEV $_{1}: p=0.001$; FVC: $p=0.001$; $\mathrm{FEV}_{1} / \mathrm{FVC}: p=0.001$; PEF: $p=0.012$ ). There were statistically significant differences in the chest expansion values and pulmonary function parameters of the participants in Group-C (CE: $p=0.007$; FEV $1: p=0.016$; FVC: $p=0.045$; PEF: $p=0.012$. There was no significant difference in $\mathrm{FEV}_{1} / \mathrm{FVC}: p=0.235$ (Table 3).

Comparison of the mean differences in chest expansion (CE) values and pulmonary function parameters across the three groups.

One-way ANOVA was used for this analysis, statistically significant differences were observed for $\mathrm{FEV}_{1}: p=0.002$; FVC: $p=0.029$; FEV $/$ FVC: $p=0.007$ and PEF: $p=0.040$. No statistically significant difference was seen in the chest expansion values ( $p=0.405)$ (Table 4$)$.

Least significant difference (LSD), post-hoc analysis shows that the significant difference for $\mathrm{FEV}_{1}, \mathrm{FVC}$ and $\mathrm{FEV}_{1} / \mathrm{FVC}$ lies between Group-A and Group-B (Table 5).

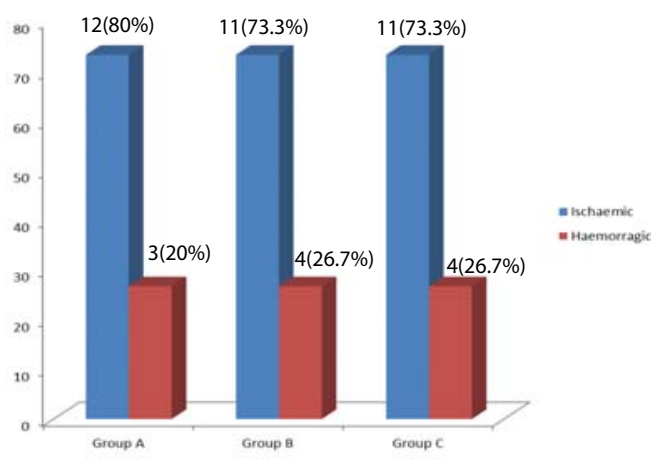

Figure 3: Bar-Chart of ischaemic and haemorrhagic strokes in each group.

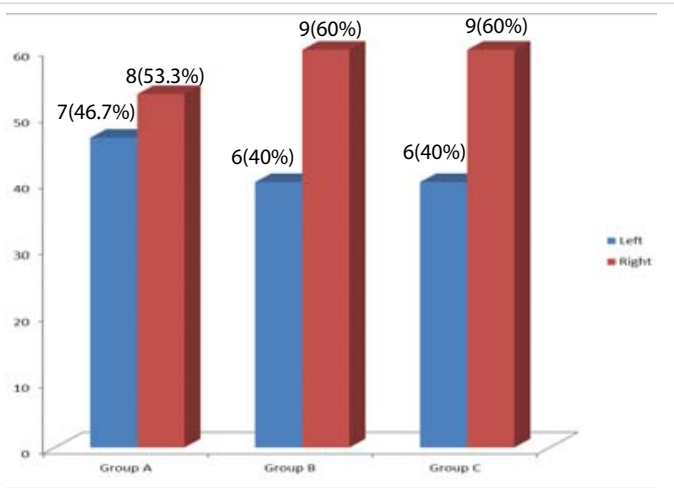

Figure 4: Bar-Chart of the paretic side of stroke affectation in each group. 
Table 2: Demographic characteristics of the participants.

\begin{tabular}{|c|c|c|c|c|c|}
\hline Variables & Group $A \bar{x} \pm$ SD $N=15$ & Group B $\bar{x} \pm$ SD N=15 & Group C $\bar{x} \pm$ SD N=15 & F - value & $p$ - value \\
\hline Age (Years) & $56.20+10.39$ & $59.27+12.98$ & $58.20+10.12$ & 0.572 & 0.569 \\
\hline Weight (Kg) & $69.13+10.79$ & $75.75+10.59$ & $72.75+12.19$ & 0.185 & 0.832 \\
\hline Height (m) & $1.71+0.07$ & $1.71+0.07$ & $1.67+0.92$ & 1.298 & 0.284 \\
\hline $\mathrm{BMI}\left(\mathrm{Kg} / \mathrm{m}^{2}\right)$ & $23.74+3.27$ & $25.92+3.31$ & $26.11+3.80$ & 0.179 & 0.837 \\
\hline
\end{tabular}

Table 3: Pre- and post - intervention comparison of the chest expansion values and pulmonary functions in the groups.

\begin{tabular}{|c|c|c|c|c|}
\hline Variables & Pre-training $\bar{x} \pm$ SD & Post-training $\bar{x} \pm$ SD & $\mathrm{t}$ - value & $p$ - value \\
\hline \multicolumn{5}{|c|}{ GROUP A } \\
\hline $\mathrm{CE}(\mathrm{cm})$ & $2.18+0.94$ & $2.78+1.20$ & -4.375 & $0.001^{*}$ \\
\hline $\mathrm{FEV}_{1}(\mathrm{~L})$ & $1.25+0.68$ & $1.44+0.76$ & -3.621 & $0.003^{*}$ \\
\hline $\mathrm{FVC} \mathrm{(L)}$ & $1.87+0.92$ & $1.97+0.92$ & -2.561 & $0.023^{*}$ \\
\hline $\mathrm{FEV}_{1} / \mathrm{FVC}$ & $0.65+0.13$ & $0.72+0.15$ & -4.252 & $0.001^{*}$ \\
\hline $\operatorname{PEF}(\mathrm{L} / \mathrm{s})$ & $2.39+1.35$ & $2.78+1.62$ & -3.309 & $0.005^{*}$ \\
\hline \multicolumn{5}{|c|}{ GROUP B } \\
\hline $\mathrm{CE}(\mathrm{cm})$ & $2.15+0.98$ & $3.38+1.20$ & -5.848 & $0.001^{*}$ \\
\hline $\mathrm{FEV}_{1}(\mathrm{~L})$ & $2.01+1.01$ & $2.94+1.25$ & -6.189 & $0.001^{*}$ \\
\hline FVC (L) & $2.50+1.14$ & $3.25+1.27$ & -5.668 & $0.001^{*}$ \\
\hline $\begin{array}{l}\mathrm{FEV}_{1} / \mathrm{FVC} \\
\mathrm{PEF}(\mathrm{L} / \mathrm{s})\end{array}$ & $\begin{array}{l}0.79+0.10 \\
3.21+1.27\end{array}$ & $\begin{array}{l}0.89+0.89 \\
4.16+1.85\end{array}$ & $\begin{array}{l}-4.159 \\
-2.887\end{array}$ & $\begin{array}{l}0.001^{*} \\
0.012^{*}\end{array}$ \\
\hline \multicolumn{5}{|c|}{ GROUP C } \\
\hline $\mathrm{CE}(\mathrm{cm})$ & $2.32+0.71$ & $3.18+1.28$ & -3.170 & $0.007^{*}$ \\
\hline $\mathrm{FEV}_{1}(\mathrm{~L})$ & $1.76+0.82$ & $2.23 \pm 1.14$ & -2.750 & $0.016^{*}$ \\
\hline $\mathrm{FVC}(\mathrm{L})$ & $2.29+1.24$ & $2.90+1.69$ & -2.196 & $0.045^{\star}$ \\
\hline $\mathrm{FEV}_{1} / \mathrm{FVC}$ & $0.76+0.15$ & $0 .-80 \pm 0.16$ & -1.241 & 0.235 \\
\hline $\operatorname{PEF}(\mathrm{L} / \mathrm{s})$ & $3.48+1.51$ & $4.22+1.60$ & -2.885 & $0.012^{*}$ \\
\hline
\end{tabular}

Table 4: Comparison of the mean differences in chest expansion values and pulmonary function across the three groups.

\begin{tabular}{|c|c|c|c|c|c|}
\hline Variables & Group $A \bar{x} \pm S D$ & Group B $\bar{x} \pm$ SD & Group C $\bar{x} \pm$ SD & F-value & $p$ - value \\
\hline \multicolumn{6}{|l|}{ PRE-TRAINING } \\
\hline CE $(\mathrm{cm})$ & $2.18+0.94$ & $2.15+0.98$ & $2.32+0.71$ & 0.428 & 0.655 \\
\hline $\mathrm{FEV}_{1}(\mathrm{~L})$ & $1.25+0.68$ & $2.01+1.01$ & $1.76+0.82$ & 1.350 & 0.270 \\
\hline $\mathrm{FVC}(\mathrm{L})$ & $1.87 \pm 0.92$ & $2.50+1.14$ & $2.29 \pm 1.24$ & 0.356 & 0.703 \\
\hline $\mathrm{FEV}_{1} / \mathrm{FVC}$ & $0.65+0.13$ & $0.79+0.10$ & $0.76+0.15$ & 1.617 & 0.211 \\
\hline PEF (L/s) 4TH WEEK POST-INTERVENTION & $2.39+1.35$ & $3.21+1.27$ & $3.48+1.51$ & 3.179 & 0.052 \\
\hline CE $(\mathrm{cm})$ & $2.78 \pm 1.20$ & $3.38 \pm 1.20$ & $3.18 \pm 1.28$ & 0.924 & 0.405 \\
\hline $\mathrm{FEV}_{1}(\mathrm{~L})$ & $1.44 \pm 0.76$ & $2.94 \pm 1.25$ & $2.23 \pm 1.14$ & 7.373 & $0.002^{*}$ \\
\hline FVC (L) & $1.97 \pm 0.92$ & $3.28 \pm 1.27$ & $2.90 \pm 0.89$ & 3.842 & $0.029^{*}$ \\
\hline $\mathrm{FEV}_{1} / \mathrm{FVC}$ & $0.72 \pm 0.15$ & $0.89 \pm 0.89$ & $0.80 \pm 0.16$ & 5.661 & $0.007^{*}$ \\
\hline PEF (L/s) & $2.78 \pm 1.61$ & $4.16 \pm 1.85$ & $4.22 \pm 1.60$ & 3.469 & $0.040^{*}$ \\
\hline
\end{tabular}

Table 5: Post-hoc analysis (LSD) across the groups.

\begin{tabular}{|c|c|c|c|c|}
\hline Variables & Group Status (I) & Group Status (J) & Mean difference (df) (l-J) & $p$ - value \\
\hline \multirow{6}{*}{$\mathrm{FEV}_{1}(\mathrm{~L})$} & \multirow{2}{*}{ Group A } & Group B & -1.501 & $0.001^{*}$ \\
\hline & & Group C & -0.783 & 0.124 \\
\hline & \multirow{2}{*}{ Group B } & Group A & 1.501 & $0.001^{*}$ \\
\hline & & Group C & 0.719 & 0.17 \\
\hline & \multirow{2}{*}{ Group C } & Group A & 0.783 & 0.124 \\
\hline & & Group B & -0.719 & 0.17 \\
\hline \multirow{6}{*}{$\mathrm{FVC}(\mathrm{L})$} & \multirow{2}{*}{ Group A } & Group B & -1.309 & $0.027^{*}$ \\
\hline & & Group C & -0.931 & 0.147 \\
\hline & \multirow{2}{*}{ Group B } & Group A & -1.309 & $0.027^{*}$ \\
\hline & & Group C & 0.379 & 0.718 \\
\hline & \multirow{2}{*}{ Group C } & Group A & 0.931 & 0.147 \\
\hline & & Group B & -0.379 & 0.718 \\
\hline \multirow{6}{*}{ FEV1/FVC } & \multirow{2}{*}{ Group A } & Group B & -0.167 & $0.005^{*}$ \\
\hline & & Group C & -0.78 & 0.268 \\
\hline & \multirow{2}{*}{ Group B } & Group A & 0.167 & $0.005^{*}$ \\
\hline & & Group C & 0.887 & 0.186 \\
\hline & \multirow{2}{*}{ Group C } & Group A & 0.78 & 0.268 \\
\hline & & Group B & -0.887 & 0.186 \\
\hline
\end{tabular}




\section{Discussion}

The specific aim of this study was to determine the comparative efficacy of inspiratory, expiratory, and combined respiratory muscle training on the pulmonary functions and chest expansion in acute stroke survivors. There were significant improvements in the chest expansion and pulmonary functions $\left(\mathrm{FEV}_{1}, \mathrm{FVC}, \mathrm{FEV}_{1} / \mathrm{FVC}\right.$, and $\left.\mathrm{PEF}\right)$ following inspiratory muscle training. This is like findings recorded after inspiratory muscle training in patients with chronic obstructive pulmonary disease (COPD) and myasthenia gravis [45,46], and they demonstrated increased chest expansion after inspiratory muscle training. Interestingly, Sahin [47] reported a positive correlation between chest expansion and maximal inspiratory pressure (MIP) in patients with ankylosing spondylitis. Moreover, displacement of the ribcage in the lateral outward direction during inspiration in normal subjects using an automatic motion analyzer was greater than the displacement during tidal breathing [48]. The lateral movement of the ribcage at the level of the fourth rib during inspiration was increased in the neutral position using electromagnetic tracking device in healthy subjects [49]. Thus, these findings support the use of a resistive-inspiratory muscle trainer as an effective chest expansion method for patients following an acute stroke [38]. Accordingly, expiratory muscle training has the potential to improve respiratory function, as well as swallowing disorders, thereby improving overall treatment of stroke patients. In this study, there were significant improvements in the chest expansion and pulmonary functions $\left(\mathrm{FEV}_{1}, \mathrm{FVC}, \mathrm{FEV}_{1} / \mathrm{FVC}\right.$, and PEF) following expiratory muscle training. Chiara [50] reported an increased in the maximal expiratory pressure and the peak expiratory flow following expiratory muscle training in patients with multiple sclerosis and concluded that expiratory muscle strength training is a viable tool to enhance the strength of the respiratory muscles. This improvement was attributed to the neural changes occurring in the motor command, descending drive, muscle activation, motor unit recruitment and sensory feedbacks [51,52]. Other studies like this present study reported significant gains in PEF, FVC, $\mathrm{FEV}_{1}$, total lung capacity and maximal ventilation following expiratory muscle strength training in both patient population and healthy subjects [53-58]. The study of Kuo [59] concluded that both 5 days per week and 3 days per week of expiratory muscle strength training effectively enhance respiratory muscle strength and improve mobility construct measured by PDQ-39 in patients with Parkinson disease. There was significant difference in the chest expansion and pulmonary parameters of $\mathrm{FEV}_{1}, \mathrm{FVC}$, and PEF but no significant difference in $\mathrm{FEV}_{1} / \mathrm{FVC}$ following combined respiratory muscle training. A similar study investigated the effects of breathing exercise on 40 stroke patients. They divided the subjects into three groups - an exercise group with feedback breathing exercise, an exercise group combined with inspiratory muscle training and expiratory muscle training, and a control group, 3 times a week for 4 weeks. The results showed a marked improvement in thoracic size and pulmonary functions in the exercise group combined with inspiratory muscle training and expiratory muscle training than in other groups [60]. Another study has reported that training of respiratory muscles in patients with cervical spine injury using the combined respiratory muscle methods increased the strength of the respiratory muscles, and increased FVC and FEV 1 [61]. Explanations as to the lack of increase in $\mathrm{FEV}_{1} / \mathrm{FVC}$ as seen in this study in the presence of increased MEP are limited. First, above a certain transpulmonary pressure at a given volume, flow does not increase with driving pressure [62]. It is not the effort or MEP, but the elastic recoil of the lung, determined by lung volumes and properties of lung tissue, which governs the maximal expiratory flow [62]. Second, the intrinsic state of the lung may influence expiratory flow. In this study, expiratory muscle training was found to be most effective for all the pulmonary function parameters and chest expansion across the three groups. A study by Moon [63] affirmed that expiratory muscle strength training (EMST) is an effective intervention for impaired swallowing function in acute stroke patients with dysphagia when compared with the control. It was concluded that EMST has the potential to improve respiratory functions, as well as swallowing disorders thereby improving overall treatment of stroke patients. The use of a resistive-expiratory muscle trainer in this study effectively trained both expiratory muscles and inspiratory muscles at a given resistance over the course of the trial than when compared to other training methods used in this study. The inspiratory muscle trainer selectively and effectively trained the inspiratory muscles without much effect on the expiratory muscles while the breather trainer (used for combined respiratory muscle training in this study) has a more pronounced effect on the inspiratory muscles than on the expiratory muscles at a given resistance over the course of the study.

\section{Conclusion}

Findings of this study showed that inspiratory muscle training, expiratory muscle training and combined respiratory muscle training have beneficial effects in improving the chest expansion and pulmonary function parameters $\left(\mathrm{FEV}_{1}, \mathrm{FVC}\right.$, $\mathrm{FEV}_{1} / \mathrm{FVC}$, and PEF) in acute stroke survivors. However, expiratory muscle training is the most beneficial clinically in improving the chest expansion and pulmonary function parameters in acute stroke survivors.

Limitation: The major limitation encountered during this study was time limit.

Implications for further studies: The study should be carried out for a longer duration of at least six weeks.

\section{References}

1. Rabelo M, Nunes GS, da Costa Amante NM, de Noronha M, FachinMartins E. Reliability of muscle strength assessment in chronic 
poststroke hemiparesis: a systematic review and meta-analysis. Top Stroke Rehabil 2016; 23: 26-36.

PubMed: https://pubmed.ncbi.nlm.nih.gov/26243549/

2. Gomes-Neto M, Saquetto MB, Silva CM, Carvalho VO, Ribeiro N, et al Effects of respiratory muscle training on respiratory function, respiratory muscle strength, and exercise tolerance in patients poststroke: a systematic review with meta-analysis. Arch Phy Med Rehabil 2016;1:1-8. PubMed: https://pubmed.ncbi.nlm.nih.gov/27216224/

3. Harik-Khan RI, Wise RA, Fozard JL Determinants of maximal inspiratory pressure. The Baltimore Longitudinal Study of Aging. Am J Respir Crit Care Med. 1998; 158: 1459-1464.

PubMed: https://pubmed.ncbi.nlm.nih.gov/9817693/

4. Harraf F, Ward K, Man W, Rafferty G, Mills K, et al. Transcranial magnetic stimulation study of expiratory muscle weakness in acute ischaemic stroke. Neurology. 2008; 71: 2000 -2007.

PubMed: https://pubmed.ncbi.nlm.nih.gov/19064882/

5. Lanini B, Bianchi R, Romagnoli I. Chest wall kinematics in patients with hemiplegia. Am J Respir Criti Care Med. 2003; 168: 109-113. PubMed: https://pubmed.ncbi.nlm.nih.gov/12714347/

6. Ward K, Seymour J, Steier J, Jolley CJ, Polkey MI, et al. Acute ischaemic hemispheric stroke is associated with impairment of reflex in addition to a voluntary cough. Eur Respir J. 2010; 36: 1383-1390. PubMed: https://pubmed.ncbi.nlm.nih.gov/20413536/

7. Polese JC, Pinheiro MB, Faria CD, Britto RR, Parreira VF, et al. The strength of the respiratory and lower limb muscles and functional capacity in chronic stroke survivors with different physical activity levels. Braz J Phy Ther. 2013; 17: 487-493.

PubMed: https://pubmed.ncbi.nlm.nih.gov/24173350/

8. Xiao $\mathrm{Y}$, Luo $\mathrm{M}$, Wang $\mathrm{J}$, Luo $\mathrm{H}$. Inspiratory muscle training for the recovery of function after stroke (review). Cochrane Database Systema Rev. 2012; 5: 1-27.

PubMed: https://pubmed.ncbi.nlm.nih.gov/22592740/

9. Almeida IC, Clementino AC, Rocha EH, Brandão DC, Andrade $A D$. Effects of hemiplegy on pulmonary function and diaphragmatic dome displacement. Respir Physiol Neurobiolol. 2011; 178: 196-201. PubMed: https://pubmed.ncbi.nlm.nih.gov/21679778/

10. Pollock RD, Rafferty GF, Moxham J, Kalra L. Respiratory muscle strength and training in stroke and neurology: a systematic review. Int J Stroke. 2013; 8: 124-130. PubMed: https://pubmed.ncbi.nlm.nih. gov/22568454/

11. Jandt SR, Caballero RM, Ju'nior LA, Dias AS. Correlation between trunk control, respiratory muscle strength and spirometry in patients with stroke: an observational study. Physiother Res Int. 2011; 16: 218224. PubMed: https://pubmed.ncbi.nlm.nih.gov/21157882/

12. Khedr EM, El Shinawy O, Khedr T, Abdelazizali Y, Awad EM Assessment of corticodiaphragmatic pathway and pulmonary function in acute ischaemic stroke patients. Eur J Neurol. 2000; 7: 323-330. PubMed: https://pubmed.ncbi.nlm.nih.gov/10886317/

13. Tomczak CR, Jelani A, Haennel RG. Cardiac reserve and pulmonary gas exchange kinetics in patients with stroke. Stroke. 2008; 39: 3102-3106. PubMed: https://pubmed.ncbi.nlm.nih.gov/18703810/

14. MacKay-Lyons MJ, Makrides L. Cardiovascular stress during a contemporary stroke rehabilitation program: is the intensity adequate to induce a training effect? Arch Phy Med Rehabil 2002; 83: 1378-1383. PubMed: https://pubmed.ncbi.nlm.nih.gov/12370872/

15. Billinger SA, Taylor JM, Quaney BM. Cardiopulmonary response to exercise testing in people with chronic stroke: a retrospective study. Stroke Res Treatment. 2012; 20: 987637.

PubMed: https://pubmed.ncbi.nlm.nih.gov/21961083/

16. Hannawi Y, Hannawi B, Rao CPV, Suarez JI, Bershad EM. Strokeassociated pneumonia: major advances and obstacles. Cerebrovasc Dis. 2013; 35: 430-443.

PubMed: https://pubmed.ncbi.nlm.nih.gov/23735757/
17. Finlayson O, Kapral M, Hall R, Asllani E, Selchen D, et al. Risk factors, inpatient care, and outcomes of pneumonia after ischaemic stroke. Neurology. 2011; 77: 1338-1345.

PubMed: https://pubmed.ncbi.nlm.nih.gov/21940613/

18. Fontana GA, Lavorini F. Cough motor mechanisms. Respir Physiol Neurobiol J. 2006; 152: 266-281.

PubMed: https://pubmed.ncbi.nlm.nih.gov/16600697/

19. Widdicombe JG, Addington WR, Fontana GA, Stephens RE. A voluntary and reflex cough and the expiration reflex; implications for aspiration after stroke. Pulmon Pharmacol Therapeut. 2011; 24: 312-317. PubMed: https://pubmed.ncbi.nlm.nih.gov/21338708/

20. Teixeira-Salmela LF, Parreira VF, Britto RR, Brant TC, Inacio EP, et al. Respiratory pressures and thoracoabdominal motion in communitydwelling chronic stroke survivors. Arch Phy Med Rehabil 2005; 86 1974-1978.

PubMed: https://pubmed.ncbi.nlm.nih.gov/16213241/

21. Sutbeyaz ST, Koseoglu F, Inan L, Coskun O. Respiratory muscle training improves cardiopulmonary function and exercise tolerance in subjects with subacute stroke: a randomized controlled trial. Clin Rehabil 2010; 24: 240-250.

PubMed: https://pubmed.ncbi.nlm.nih.gov/20156979/

22. Pryor JA, Prasad AS. Physiotherapy for respiratory and cardiac problems: adults and paediatrics (5th Edition). Elsevier Health Sciences, USA. 2008.

23. Kelly JO, Kilbreath SL, Davis GM. Cardiorespiratory fitness and walking ability in subacute stroke patients. Arch Phys Med Rehabil 2003; 84: 1780-1785.

PubMed: https://pubmed.ncbi.nlm.nih.gov/14669183/

24. Skinner JS. Exercise testing and exercise prescription for special cases: theoretical basis and clinical application (3rd Edition). Lippincott Williams \& Wilkins, Philadelphia, USA. 2005.

25. CourbonA, CalmelsP, RocheF, RamasJ, RimaudD, etal. Therelationship between maximal exercise capacity and walking capacity in adult hemiplegic stroke patients. Am J Phy Med Rehabil 2006; 85: 436-442. PubMed: https://pubmed.ncbi.nIm.nih.gov/16628151/

26. Moodie LH, Reeve JC, Vermeulen N, Elkins MR. Inspiratory muscle training to facilitate weaning from mechanical ventilation: Protocol for a systematic review. Bri Med Res Notes. 2011; 4: 283.

PubMed: https://pubmed.ncbi.nlm.nih.gov/21835031/

27. Britto RR, Rezende NR, Marinho KC, Torres JL, Parreira VF, et al Inspiratory muscular training in chronic stroke survivors: a randomized controlled trial. Arch Phy Med Rehabil 2011; 92: 184-190.

PubMed: https://pubmed.ncbi.nlm.nih.gov/21272713/

28. St Croix CM, Morgan BJ, Wetter TJ. Fatiguing inspiratory muscle work causes reflex sympathetic activation in humans. J Physiol. 2010; 529 : 493-504. PubMed: https://pubmed.ncbi.nlm.nih.gov/11101657/

29. Babcock MA, Pegelow DF, McClaran SR. The contribution of diaphragmatic power output to exercise-induced diaphragm fatigue. J Appl Physiol. 1995; 78: 1710-1719.

PubMed: https://pubmed.ncbi.nlm.nih.gov/7649904/

30. Beckerman M, Magadle R, Weiner $M$. The effects of 1 year of specific inspiratory muscle training in patients with COPD. Chest. 2005; 128 : 3177-3182. PubMed: https://pubmed.ncbi.nlm.nih.gov/16304259/

31. Inzelberg R, Peleg N, Nisipeanu P, Magadle R, Carasso RL, et al. Inspiratory muscle training and the perception of dyspnoea in Parkinson's disease. Can J Neurological Sci. 2005; 32: 213-217. PubMed: https://pubmed.ncbi.nlm.nih.gov/16018157/

32. McConnell AK. Respiratory Muscle Training: Theory and Practice. Edinburgh, Churchill Livingstone/Elsevier. 2013.

33. Kyo Chul S, Hyun Min L, Hyeon Ae K. The effects of the combination of inspiratory diaphragm exercise and expiratory Pursed-Lip breathing exercise on pulmonary functions of stroke patients. J Phy Ther Sci. 2013; 25: 241-244. 
34. Hsiao SF, Wu YT, Wu HD. Comparison of effectiveness of pressure threshold and targeted resistance devices for inspiratory muscle training in patients with the chronic obstructive pulmonary disease. $J$ Formosan Med Associat. 2003; 102: 240-245.

PubMed: https://pubmed.ncbi.nlm.nih.gov/12833187/

35. Ray AD, Udhoji S, Mashtare TL. A combined inspiratory and expiratory muscle-training program improves respiratory muscle strength and fatigue in multiple sclerosis. Arch Phy Med Rehabil 2013; 94: 1964-1970. PubMed: https://pubmed.ncbi.nlm.nih.gov/23714277/

36. Weiner $P$, Magadle R, Beckerman M. Comparison of specific expiratory, inspiratory, and combined muscle training programs in COPD. Chest 2003; 124: 1357-1364.

PubMed: https://pubmed.ncbi.nlm.nih.gov/14555566/

37. Cohen J. Power primer. Psychological bulletin. 1992; 112: 155-159. PubMed: https://pubmed.ncbi.nlm.nih.gov/19565683/

38. Kim K, Fell DW, Lee JH. Feedback respiratory training to enhance chest expansion and pulmonary function in chronic stroke: a doubleblind randomized controlled study. J Phy Ther Sci. 2011; 23: 75-79.

39. Viitanem JV, Kautiainen $\mathrm{H}$, Suni J. The relative value of spinal and thoracic mobility measurement in ankylosing spondylitis. Scandinavian J Rheumatol. 1995; 24: 94-97.

PubMed: https://pubmed.ncbi.nlm.nih.gov/7747150/

40. Evans JA, Whitelaw WA. The assessment of maximal respiratory mouth pressures in adults. Respir Care. 2009; 54: 1348-1359. PubMed: https://pubmed.ncbi.nlm.nih.gov/19796415/

41. Centers for Disease Control and Prevention. Growing stronger-strength training for the older adults. 2017; http://healthyliving.azcentral.com

42. Kim JH, Park JH, Yim J. Respiratory exercise in stroke patients. Med Sci Monitor. 2014; 20: 2543-2549.

PubMed: https://pubmed.ncbi.nlm.nih.gov/25488849/

43. Kulnik ST, Rafferty GF, Birring SS, Moxham J, Kalra L. A pilot study of respiratory muscle training to improve the incidence of pneumonia in acute stroke: study protocol for a randomized controlled trial. Trials. 2014; 15: 123.

PubMed: https://pubmed.ncbi.nlm.nih.gov/24725276/

44. Messaggi-Sartor M, Guillen-Solà A, Depolo M, Duarte E, Rodríguez $D A$, et al. Inspiratory and expiratory muscle training in subacute stroke: A randomized clinical trial. Neurology. 2015; 85: 564-572.

PubMed: https://pubmed.ncbi.nlm.nih.gov/26180145/

45. Minoguchi $H$, Shibuya $M$, Miyagawa T. Cross-over comparison between respiratory muscle stretch gymnastics and inspiratory muscle training. Internal Med. 2002; 41: 805-812.

PubMed: https://pubmed.ncbi.nlm.nih.gov/12413000/

46. Fregonezi GA, Resqueti VR, Guell R, Prada J, Casan P. Effects of 8-week, interval-based inspiratory muscle training and breathing retraining in patients with generalized myasthenia gravis. Chest. 2005; 128: $1524-1530$.

PubMed: https://pubmed.ncbi.nlm.nih.gov/16162753/

47. Sahin G, Calikoglu M, Ozge C. Respiratory muscle strength but not BASFI score relates to diminished chest expansion in ankylosing spondylitis. Clin Rheumatol. 2004; 23: 199-202.

PubMed: https://pubmed.ncbi.nlm.nih.gov/15168144/

48. De Groote A, Wantier M, Cheron G. Chest wall motion during tidal breathing. J Appl Physiol. 1997; 83: 1531-1537. PubMed: https://pubmed.ncbi.nlm.nih.gov/9375316/
49. Lee LJ, Chang AT, Coppieters MW. Changes in sitting posture induce multiplanar changes in chest wall shape and motion with breathing. Respir Physiol Neurobiol. 2010; 170: 236-245. PubMed: https://pubmed.ncbi.nlm.nih.gov/20083236/

50. Chiara T, Martin DA, Davenport PW, Bolser DC. Expiratory muscle strength training in persons with multiple sclerosis having mild to moderate disability: Effect on maximal expiratory pressure, pulmonary function, and maximal voluntary cough. Arch Phy Med Rehabil 2006; 87: 468-473.

PubMed: https://pubmed.ncbi.nlm.nih.gov/16571384/

51. McComas A. Human neuromuscular adaptations that accompany changes in activity. Med Sci Sports Exercise. 1994; 26: 1498-1509. PubMed: https://pubmed.ncbi.nlm.nih.gov/7869885/

52. Duchateau J, Enoka R. Neural adaptations with chronic activity patterns in able-bodied humans. Am J Phy Med Rehabil 2002; 81: 17-27. PubMed: https://pubmed.ncbi.nlm.nih.gov/12409808/

53. Olgiati R, Girr A, Hugi L, Haegi V. Respiratory muscle training in multiple sclerosis: a pilot study. Swiss Arch Neurol Psychiatry Psychother. 1989; 140: 46-50.

PubMed: https://pubmed.ncbi.nlm.nih.gov/2467371/

54. Biering-Sorensen F, Lehmann Knudsen J, Schmidt A, Bundgaard A, Christensen I. Effect of respiratory training with a mouth-nose-mask in tetraplegics. Paraplegia. 1991; 29: 113-139.

PubMed: https://pubmed.ncbi.nlm.nih.gov/2023775/

55. Weiner $P$, Gross D, Meiner Z. Respiratory muscle training in patients with moderate to severe myasthenia gravis. Canadian J Neurological Sci. 1998; 25: 236-241.

PubMed: https://pubmed.ncbi.nlm.nih.gov/9706726/

56. Liaw MY, Lin MC, Cheng PT, Wong M, Tang F. Resistive inspiratory muscle training: its effectiveness in patients with acute complete cervical cord injury. Arch Phy Med Rehabil 2000; 81: 752-756. PubMed: https://pubmed.ncbi.nlm.nih.gov/10857519/

57. Wang T, Wang Y, Tang F, Lin K, Lien I. Resistive inspiratory muscle training in sleep-disordered breathing of traumatic tetraplegia. Arch Phy Med Rehabil 2002; 83:491-496.

PubMed: https://pubmed.ncbi.nlm.nih.gov/11932850/

58. Klefbeck B, Nedjad JH. Effect of inspiratory muscle training in patients with multiple sclerosis. Arch Phy Med Rehabil 2003; 84: 994-999. PubMed: https://pubmed.ncbi.nlm.nih.gov/12881823/

59. Kuo YC, Chan J, Wu YP, Bernard JR, Liao YH. Effect of expiratory muscle strength training intervention on the maximum expiratory pressure and quality of life of patients with Parkinson's disease. Neuro Rehabil 2017; 41: 219-226.

PubMed: https://pubmed.ncbi.nlm.nih.gov/28527233/

60 . Jeonhyeong $L$. The effect of pulmonary function in the stroke patients after feedback breathing exercise. Daegu University. 2008.

61. Ehrlich M, Manns PJ, Poulin C. Respiratory training for a person with $\mathrm{C}_{3}-\mathrm{C}_{4}$ tetraplegia. Australian J Physiother. 1999; 45: 301-307.

PubMed: https://pubmed.ncbi.nlm.nih.gov/11676779/

62. Hyatt R. Expiratory flow limitation. J Appl Physiol. 1983; 55: 1-7. PubMed: https://pubmed.ncbi.nlm.nih.gov/6350246/

63. Moon JH, Jung J, Won YS, Cho HY, Cho K. Effects of expiratory muscle strength training on swallowing function in acute stroke patients with dysphagia. J Phy Ther Sci. 2017; 29: 609-612. PubMed: https://pubmed.ncbi.nlm.nih.gov/28533594/ 